\title{
Proposal of Risk Tolerance Factor for Optimizing Risk Management, based on the system context - Nanosatellite Case Studies
}

Benjamim Monteiro ( $\sim$ benjamimguerra@gmail.com )

Federal University of Rio Grande do Norte

Giuliani Garbi

National Institute for Space Research

Francisco Vidal

Federal University of Rio Grande do Norte

Manoel de Carvalho

National Institute for Space Research

Alessandra Rodrigues

National Institute for Space Research

\section{Research Article}

Keywords: risk management, acquisition, CubeSat, Risk Tolerance Factor

Posted Date: March 7th, 2022

DOI: https://doi.org/10.21203/rs.3.rs-1319742/v1

License: (9) This work is licensed under a Creative Commons Attribution 4.0 International License. Read Full License 


\section{Abstract}

The paper present and implement a generic methodology for optimizing the risk management of low-cost systems with a lean schedule and brief operating time. Therefore, a proposed approach uses a correction factor (RTF - Risk Tolerance Factor) in the qualitative risk management stage. To analyze the application of the RTF approach, two case studies, ARMADILLO and CONASSAT, are presented. It was observed that the use of the RTF approach provided the mitigation of a minor number of risks in the planning stage of risk management in CubeSats projects. The advantage of the RTF approach is that the project team will carry out more straightforward mitigation plans for qualified risks. It is concluded that the approach met the objective of providing the team members with information about which risk scenario the mission is allocated in at the beginning of the mission design process. However, it does not exclude the possibility of later application of the tool.

\section{Introduction}

A space system is composed of a ground segment, with its command and tracking antennas; space segment, this being the artificial satellite; launcher segment, with its launch infrastructure; and user segment, which will use the data provided by the artificial satellite ${ }^{1,2}$. Space systems and their segments are developed using the Systems Engineering (SE) approach and the system life cycle, often composed of phases or stages ${ }^{3}$.

The focus of this paper will be the space segment, these artificial satellites being classified according to their mass as large satellites (those with a mass greater than one thousand kilograms), medium satellites (those with a mass between one hundred and one thousand kilograms), and small satellites (those with a mass less than one hundred kilograms) ${ }^{4}$. More specifically, the CubeSats stand out when it comes to small satellites, which are satellites formed by units or combinations of cubic units with a 10-cm edge. CubeSats were proposed in 1999 by professors Jordi Puig-Suari and Bob Twiggs from California Polytechnic State University (Cal-Poly) and Stanford University ${ }^{5}$.

Initially, CubeSats were developed for academic applications and technology demonstrations, with the characteristic of being low-cost systems (hundreds of thousands of dollars), with a lean schedule, between 12 months and 18 months, and short operating time, about 12 months in low orbit ${ }^{6,7}$. Currently, the miniaturization of electrical/electronic components and the use of off-the-shelf devices and equipment (COTS) enable CubeSats in operational missions that previously could only be performed by medium or large satellites ${ }^{8}$.

However, for Cubesats to be used in operational missions, their development must minimally follow the $\mathrm{SE}^{9}$. The use of $\mathrm{SE}$ in CubeSats projects provides an approach that helps in conducting the project and ensures the validation and verification of system requirements ${ }^{10}$. 
A discipline required by $S E$ is risk management, which is responsible for identifying, qualifying, quantifying, tracking, and mitigating system risks ${ }^{3}$.

Gamble and Bouwmeester (2016) ${ }^{11}$ conducted a study on risk management with 114 CubeSat developers. It was found that $55 \%$ of developers use risk management, while $45 \%$ have not used it so far. Reasons for not using management were pointed out: firstly, a lean development schedule for CubeSats and; secondly, the lack of experience of the development teams involved.

Thus, according to Brumbaugh and Lishtsey $(2012,2013)^{12,13}$, to encourage the use of risk management in developing CubeSats to meet the characteristics of low cost, short schedule, and operating time of about 12 months, adaptation of the traditional risk management discipline is needed.

The literature presents two fronts to solve the demand for adaptation of traditional risk management: the first involves the automation of risk management in Cubesats, as proposed by Gamble and Lightsey $(2014)^{14}$.

The second front involves simplifying the steps and activities of traditional risk management to be used in the development of CubeSats, as proposed by Brumbaugh and Lightsey $(2012,2013){ }^{12,13}$ and Yassi and Sousa $(2017)^{15}$.

To adapt traditional risk management to projects of different systems with the characteristics of small satellites, this paper proposes an approach that uses a correction factor (RTF - Risk Tolerance Factor) to be used in the qualitative stage of risk management of the system. RTF is a generic approach, which, in this paper, is implemented in CubeSats projects to streamline risk management, as required by SE.

The use of the RTF approach provided greater agility in the risk management qualification stage in CubeSats projects. Furthermore, the advantage of the RTF approach is that the project team will carry out more straightforward mitigation plans for qualified risks. The RTF approach advantages are proved by the presentation of two case studies, in which qualitative risk analyses are compared between the traditional space sector risk management approach, the approach adapted to CubeSats, presented by Yassi and Sousa (2017) ${ }^{15}$, and the RTF approach, introduced in this paper.

\section{Results}

\section{ARMADILLO Case Study}

In this section, the case study of the ARMADILLO mission is presented with the application of the methodologies: DoD, risk management for CubeSats by Yassi and Sousa $(2017)^{15}$, and RTF, to analyze the risks in probability versus impact matrices.

The CubeSat ARMADILLO (Atmosphere Related Measurements and Detection of submILLimeter Objects) was a $3 U$ CubeSat developed by the SDL (Satellite Design Laboratory) of the University of Austin in Texas 
(USA).

The development of the satellite began in 2012, and the launch took place in 2019. The mission's primary objectives were: to characterize sub-millimeter-level dust and debris in-situ in LEO orbit ${ }^{13}$.

Table 1 presents the identified risks for the ARMADILLO mission, with a description of the risk, the risk type rating, the risk TAG, and the assigned probability and impact values for each risk.

Table 1

Identified risks and qualitative risk analysis for the ARMADILLO mission.13.

\begin{tabular}{|lllll|}
\hline Risk Description & Risk Type & TAG & Probability & Impact \\
\hline $\begin{array}{l}\text { Risk of non-delivery of the engineering model for the } \\
\text { competition flight committee review }\end{array}$ & Schedule & SCH & 3,98 & 3,59 \\
\hline $\begin{array}{l}\text { Failure to acquire data from the scientific mission in } \\
\text { orbit }\end{array}$ & Payload & PAY & 4,00 & 3,00 \\
\hline Being unable to communicate with the satellite & Spacecraft & SP-1 & 2,19 & 3,07 \\
\hline Being unable to acquire satellite data & Spacecraft & SP-2 & 2,05 & 3,14 \\
\hline $\begin{array}{l}\text { Inability to achieve industrial quality standards } \\
\text { Loss of human knowledge and experience }\end{array}$ & Spacecraft & SP-3 & 1,09 & 2,09 \\
\hline $\begin{array}{l}\text { Mission to have a substantial increase in cost to be } \\
\text { able to be taken forward }\end{array}$ & Cost & PER & 3,95 & 2,56 \\
\hline
\end{tabular}

\subsection{Probability and Impact Matrix - DoD Risk Management}

With the application of the DoD methodology ${ }^{16}$ for the ARMADILLO mission, we obtained the probability versus impact matrix shown in Fig. 1:

In the DoD model of the seven risks identified in the ARMADILLO mission, one was in the acceptable zone (SP-3), five were in the mitigable zone (PER, PAY, COST, SP-1, and SP-2), and one was in the zone to be avoided $(\mathrm{SCH})$.

\subsection{Probability and Impact Matrix - Methodology for Technical Risk Management for Projects with CubeSats (Yassi and Sousa, 2017) ${ }^{15}$}

This methodology uses the concept of risk level, presented in topic 3 . By applying this methodology to the ARMADILLO mission, table 2 is obtained: 
Table 2

Identification, qualitative analysis of risks and risk level for the ARMADILLO mission of the Yassi and Sousa methodology 13,15

\begin{tabular}{|c|c|c|c|c|c|}
\hline Risk Description & Risk Type & TAG & Probability & Impact & $\begin{array}{l}\text { Risk } \\
\text { Level }\end{array}$ \\
\hline $\begin{array}{l}\text { Risk of non-delivery of the engineering } \\
\text { model for the competition flight committee } \\
\text { review }\end{array}$ & Schedule & $\mathrm{SCH}$ & 3,98 & 3,59 & 3 \\
\hline $\begin{array}{l}\text { Failure to acquire data from the scientific } \\
\text { mission in orbit }\end{array}$ & Payload & PAY & 4,00 & 3,00 & 2 \\
\hline $\begin{array}{l}\text { Being unable to communicate with the } \\
\text { satellite }\end{array}$ & Spacecraft & SP-1 & 2,19 & 3,07 & 1 \\
\hline Being unable to acquire satellite data & Spacecraft & SP-2 & 2,05 & 3,14 & 1 \\
\hline $\begin{array}{l}\text { Inability to achieve industrial quality } \\
\text { standards }\end{array}$ & Spacecraft & SP-3 & 1,09 & 2,09 & 1 \\
\hline Loss of human knowledge and experience & Personnel & PER & 3,95 & 2,56 & 2 \\
\hline $\begin{array}{l}\text { Mission to have a substantial increase in } \\
\text { cost to be able to be taken forward }\end{array}$ & Cost & COST & 3,00 & 3,50 & 2 \\
\hline
\end{tabular}

With the help of this table, it is possible to generate the probability versus impact matrix represented in Fig. 2

In this methodology of the seven risks identified in the ARMADILLO mission, one was in the low-risk zone (SP-3), five were in the medium risk zone (PER, PAY, COST, SP-1, and SP-2), and one was in the zone and high risk $(\mathrm{SCH})$.

\subsection{RTF Methodology}

The criteria for determining the tolerance factors for each type of risk identified were developed according to the criteria described below to use the RTF methodology.

Schedule risks: the smaller the schedule flexibility, the smaller the tolerance factor should be; similarly the more significant the flexibility, the greater the factor.

Payload Risks: These are risks related to the mission payload. A payload composed of COTS components and with extensive flight heritage should have a small risk tolerance, as well as a payload that does not use COTS and has no flight heritage, should have greater risk tolerance.

Spacecraft Risks are risks related to the satellite platform used in the mission. For example, a satellite composed of subsystems that use COTS and with broad flight heritage should have a small risk tolerance, just as a satellite that does not use COTS and does not have flight heritage should have a greater tolerance to risk. 
Personnel risks: the experience and training of the development team are also influencing factors for risk tolerance. The greater the training and experience, the lower the tolerance factor; the less training and experience, the greater the factor.

Cost risks: concerns the availability of financial resources to accomplish the mission. The lower the availability of resources, the lower the tolerance factor; the more significant the availability, the greater the tolerance factor.

Note that the values described herein are indicative and may vary with each organization's ability to tolerate a given risk. If an identified risk applies to more than one category, the most severe RTF must be considered.

Then, the parameters were applied to Table 1, forming Table 3, which, together with the previous risk information, also presents the RTF values obtained for each type of risk in the mission. Therefore, the risks were classified in the probability versus impact matrices and illustrated in Figs. 3 and 4.

Table 3

Identification and classification of risks following the RTF approach, ARMADILLO mission ${ }^{13}$

\begin{tabular}{|llllll|}
\hline Risk Description & Risk Type & TAG & Probability & Impact & RTF \\
\hline $\begin{array}{l}\text { Risk of non-delivery of the engineering model } \\
\text { for the competition flight committee review }\end{array}$ & Schedule & SCH & 3,98 & 3,59 & 3 \\
\hline $\begin{array}{l}\text { Failure to acquire data from the scientific } \\
\text { mission in orbit }\end{array}$ & Payload & PAY & 4,00 & 3,00 & 5 \\
\hline $\begin{array}{l}\text { Being unable to communicate with the } \\
\text { satellite }\end{array}$ & Spacecraft & SP-1 & 2,19 & 3,07 & 5 \\
\hline $\begin{array}{l}\text { Being unable to acquire satellite data } \\
\begin{array}{l}\text { Inability to achieve industrial quality } \\
\text { standards }\end{array}\end{array}$ Spacecraft & SP-2 & 2,05 & 3,14 & 5 \\
\hline $\begin{array}{l}\text { Loss of human knowledge and experience } \\
\text { Spacecraft }\end{array}$ & SP-3 & 1,09 & 2,09 & 5 \\
\hline $\begin{array}{l}\text { Mission to have a substantial increase in cost } \\
\text { to be able to be taken forward }\end{array}$ & Cost & COST & 3,00 & 3,50 & 3 \\
\hline
\end{tabular}

As two RTF values were obtained, two matrices were used, one for each determined RTF and its respective risks. These matrices can be seen in Figs. 3 and 4 .

Using the RTF methodology, of the seven risks identified in the ARMADILLO mission, three were in the acceptance zone (SP-1, SP-2, and SP-3), three were in the mitigation zone (PAY, PER, COST), and one of the risks was in the zone to be avoided $(\mathrm{SCH})$.

\subsection{Analysis of probability versus impact matrices for different methodologies - ARMADILLO case study}


By analyzing the probability versus impact matrices and the qualitative risks that should be dealt with in each methodology, it is possible to visualize the optimization of the risk management process proposed by the RTF methodology. Table 4illustrates the qualitative risks that will make up the risk mitigation plans.

Table 4

Comparisons between methodologies for the ARMADILLO mission

\section{COMPARATIVE - ARMADILLO Mission}

\begin{tabular}{|llll|}
\hline Approach & Acceptable Risks & Risks that require mitigation & Risks that must be avoided \\
\hline DoD & 1 & 5 & 1 \\
\hline Yassi \& Sousa & 1 & 5 & 1 \\
\hline RTF & 3 & 3 & 1 \\
\hline
\end{tabular}

The DoD methodology and Yassi and Sousa obtained the same treatment for the risks. The RTF methodology managed to have an increase in the number of acceptable risks by $200 \%$ (with the addition of two of them), and a $40 \%$ reduction in mitigable risks (with the decrease of two of them).

This result demonstrates that the approach can identify and classify risks and provide a scenario that is more by the organization's capabilities to accept, mitigate or avoid risk, with a smaller number of risks to be dealt with.

\section{CONASAT Case Studies}

This topic presents the case study of the CONASAT mission applying the methodologies: DoD $^{16}$, for risk management for Yassi and Sousa's CubeSats $(2017)^{15}$; and RTF, to highlight the risks identified in the probability versus impact matrix.

The CONASAT mission began in 2010 with a public notice issued by the Brazilian Space Agency (AEB). The mission's primary objective is to replace the current space segment of the SBCDA (Brazilian Environmental Data Collection System), which has been in orbit since the 1990s.

The project is in an advanced stage of development, already having the first satellite that will make up the constellation (flight and engineering model).

For the CONASAT mission, seven risks were also identified: risk of budget unavailability (TAG - COST); turnover of project members (TAG - PER); technological immaturity of the payload (TAG - PAY); discontinuity of the project (TAG - PROJ); inability to acquire satellite data (TAG - SP-1); inability to communicate between the satellite and the payload (TAG - SP-2); inability to send commands to the satellite (TAG - SP-3); 
Table 5 presents the risks identified for the CONASAT mission, with a description of the risk, the risk type classification, the risk TAG, and the assigned probability and impact values for each risk.

Table 5

Identified risks and qualitative risk analysis for the CONASAT mission

\begin{tabular}{|lllll|}
\hline Risk Description & Risk Type & TAG & Probability & Impacto \\
\hline Risk of budget unavailability & Cost & COST & 3,87 & 4,01 \\
\hline Project member turnover & Personnel & PER & 3,86 & 3,72 \\
\hline Technological immaturity of the payload & Payload & PAY & 2,19 & 3,88 \\
\hline Project discontinuity & Schedule & PROJ & 3,57 & 3,92 \\
\hline Inability to acquire satellite data & Spacecraft & SP-1 & 1,52 & 3,78 \\
\hline $\begin{array}{l}\text { Inability to communicate between satellite and } \\
\text { payload }\end{array}$ & Spacecraft & SP-2 & 2,22 & 3,56 \\
\hline Inability to send commands to the satellite & Spacecraft & SP-3 & 1,09 & 3,50 \\
\hline
\end{tabular}

\subsection{DoD Risk Management Application}

With the application of the DoD methodology ${ }^{16}$ for the CONASAT mission, the following probability versus impact matrix for the mission was obtained, as shown in Fig. 5 :

In the DoD model, of the seven risks identified for the CONASAT mission, two were in the acceptable zone (SP-1 and SP-3), two were in the mitigable zone (PAY and SP-2), and three were in the avoidable zone (PER, PROJ, and COST).

\subsection{Methodology for Technical Risk Management for Projects with CubeSats (Yassi and Sousa)}

In this methodology, the concept of risk level, presented in topic 3 , is used. When applying the methodology to the CONASAT mission, Table 6is obtained: 
Table 6

Risk level mission CONASAT Methodology for Technical Risk Management for Projects with CubeSats Yassi and Sousa methodology

\begin{tabular}{|llllll|}
\hline Risk Description & Risk Type & TAG & Probability & Impact & $\begin{array}{c}\text { Risk } \\
\text { Level }\end{array}$ \\
\hline Risk of budget unavailability & Cost & COST & 3,87 & 4,01 & 3 \\
\hline Project member turnover & Personnel & PER & 3,86 & 3,72 & 3 \\
\hline Technological immaturity of the payload & Payload & PAY & 2,19 & 3,88 & 2 \\
\hline Project discontinuity & Schedule & PROJ & 3,57 & 3,92 & 3 \\
\hline Inability to acquire satellite data & Spacecraft & SP-1 & 1,52 & 3,78 & 1 \\
\hline $\begin{array}{l}\text { Inability to communicate between satellite } \\
\text { and payload }\end{array}$ & Spacecraft & SP-2 & 2,22 & 3,56 & 2 \\
\hline Inability to send commands to the satellite & Spacecraft & SP-3 & 1,09 & 3,50 & 1 \\
\hline
\end{tabular}

With this table, it is possible to generate the probability versus impact matrix represented in Fig. 6 :

In this methodology, of the seven risks identified in the CONASAT mission, two were in the low-risk zone (SP-1 and SP-3), two were in the medium risk zone (SP-2 and PAY), and three were in the high-risk zone (PER, COST, and PROJ).

\subsection{RTF Methodology}

After defining the parameters for determining the RTF, they were applied to Table 5, giving rise to Table 7, which, together with the information previously presented on risks, also shows the RTF values obtained for each type of risk of the mission. Finally, the risks were classified in probability versus impact matrices and illustrated in Figs. 7, 8, and 9.

Table 7

Identification and classification of risks following the RTF approach, CONASAT mission

\begin{tabular}{|llllll|}
\hline Risk Description & Risk Type & TAG & Probability & Impact & RTF \\
\hline Risk of budget unavailability & Cost & COST & 3,87 & 4,01 & 4 \\
\hline Project member turnover & Personnel & PER & 3,86 & 3,72 & 3 \\
\hline Technological immaturity of the payload & Payload & PAY & 2,19 & 3,88 & 3 \\
\hline Project discontinuity & Schedule & PROJ & 3,57 & 3,92 & 5 \\
\hline Inability to acquire satellite data & Spacecraft & SP-1 & 1,52 & 3,78 & 3 \\
\hline $\begin{array}{l}\text { Inability to communicate between satellite } \\
\text { and payload }\end{array}$ & Spacecraft & SP-2 & 2,22 & 3,56 & 3 \\
\hline Inability to send commands to the satellite & Spacecraft & SP-3 & 1,09 & 3,50 & 3 \\
\hline
\end{tabular}


As more than one RTF value was obtained, three matrices were used; one for each RTF determined and its respective risks. These matrices can be seen in Figs. 7, 8, and 9.

Using the RTF methodology, of the seven risks identified in the CONASAT mission, two were in the acceptance zone (SP-1 and SP-3), four were in the mitigation zone (COST, PROJT, SP-2, and PAY), and only one of the risks was in the avoidable zone (PER).

\subsection{Analysis of different methodologies}

Analyzing the probability versus impact matrices and the quantitative risks to be dealt with in each methodology makes it possible to visualize the optimization of the risk management process proposed by the RTF methodology. Table 8 illustrates the number of risks that each methodology will deal with.

Table 8

Comparisons between methodologies for the CONASAT mission

\begin{tabular}{|llll|}
\hline \multicolumn{4}{|l|}{ COMPARATIVE - CONASAT Mission } \\
\hline Approach & Approach & Approach & Approach \\
\hline DoD & 2 & 2 & 3 \\
\hline Yassi \& Sousa & 2 & 2 & 3 \\
\hline RTF & 2 & 4 & 1 \\
\hline
\end{tabular}

The DoD methodology and Yassi and Sousa obtained the same treatment for risks. The RTF methodology managed to have an increase in the number of mitigable risks by $100 \%$ (with the addition of two of them), and a decrease in $67 \%$ of the risks to be avoided (with the decrease of two of them).

This result demonstrates that the approach can identify and classify risks and provide a scenario that is more following the organization's capabilities to accept, mitigate or avoid risk, with a smaller number of risks to be dealt with.

\section{Discussion}

The composition of the organization's scenarios to determine tolerance factors (RTF) should be comprehensive and multidisciplinary, considering the management disciplines and techniques of the SE that are required for the system's risk management plan. In addition, the RTF scale should follow the scale chosen by the system development team for the probability and impact of identified risks.

The RTF methodology is indicated to optimize the risk management of systems with low-cost characteristics, with a lean schedule and short operating time. In the case of this paper, the methodology was implemented to optimize the risk management of CubeSats projects, as presented in the CubeSat ARMADILLO and CubeSat CONASAT case studies. 
To encourage the use of the RTF methodology, the authors suggest future work: implement the RTF methodology to optimize risk management in systems from different areas and sectors, such as automotive, electro-electronics, aeronautics, among others. Furthermore, to streamline the communication process between team members, it is suggested to use the RTF methodology using internet-of-things tools and, finally, the RTF methodology to be inserted in the macro processes of identification and qualification risks. There is also the suggestion to extend the RTF methodology to the risk quantification macro process.

\section{Methods}

\section{Traditional risk management in the space sector}

The traditional risk management used in the space sector follows the DoD standard - Risk Management Guide for DoD Acquisition, applied by the National Aeronautics and Space Administration (NASA); and the ECSS-M-ST-80C standard, applied by the agency European Space Agency (ESA).

This paper presents, in detail, the standard used by NASA, as it is applied to the case studies of this research.

The DoD standard divides risk management into stages. The first involves identifying risks, often based on previous experiences, brainstorming, lessons learned from other projects, among others.

In the second stage, the risk analysis is carried out with the aid of an impact versus probability matrix, in which the risks are qualified, with the attribution of impact and probability values for each identified risk.

In the third stage, the risk mitigation plan is prepared, in which acceptance, transfer, mitigation, prevention, control, among others, are used to address the risks.

The fourth stage involves implementing the risk mitigation plan and monitoring the measures adopted to address those that have been identified.

Finally, in the fifth stage, there is the tracking of risks and their impacts during the development of the system. Identifying new risks may also occur, which must go through the entire risk management process, as shown in Fig. 10.

\section{Adapted risk management for CubeSats}

The literature highlights two initiatives among the ways to adapt risk management for systems such as nanosatellites. In the first one, the proposal is the automation of activities and steps of traditional risk management, as presented in the works of Gamble and Lightsey (2014) ${ }^{14}$ and Brumbaugh and Linghtsey $(2013)^{13}$. The second initiative optimizes the steps and processes of traditional risk management, 
according to Shah, Khan, and Asma (2012) $)^{17}$, Yassi and Sousa $(2017)^{15}$, Johnson et al. $(2017)^{18}$, and Ahn, Kim, and Choi $(2019)^{19}$.

In the case studies, this paper uses the methodology for technical risk management for projects with CubeSats, presented by Yassi and Sousa (2017), which will be described in detail in this topic.

This methodology follows four steps. The first is identifying risks, in which seven types of risks are defined for missions with CubeSats: safety, operational, technical performance, schedule, responsibility, regulation, and reasonableness. Risks are also identified as to the stage of the mission they impact, namely pre-launch, launch, and post-launch.

In the second stage, risk qualification, the methodology uses the impact versus probability matrix, based on which the "risk level" is defined, as shown in Fig. 11.

In the third stage, the definition of negotiations for each risk occurs. The methodology presents six ways to process risks: accepting, mitigating, avoiding, researching, monitoring, and transferring.

The risk management plan is prepared in the fourth and last step, including a detailed description of the system's risk management steps. The flow of activities and steps of the methodology can be seen in Fig. 12.

\section{Risk Management with Risk Tolerance Factor (RTF)}

This paper proposes a correction factor in the probability versus impact matrix, specifically in the risk qualification stage. This factor is determined according to the context in which the system is inserted, represented by a scale. In this paper, it is assumed that the scale is between 1 and 5 . An RTF of 1 represents a very low-risk tolerance factor, and an RTF of 5 represents a very high tolerance factor. The higher the RTF, the greater the system's risk tolerance; the lower the RTF, the lower the risk tolerance.

Analyzing the context in which the system fits, assigning RTF values to the identified risks is possible. Context is understood as the different degrees of tolerance that the organization can have concerning each identified risk, making it necessary to analyze the scenario in which each risk fits, optimizing the work of mitigating or avoiding risks, accelerating the risk management process, and having a more agile risk treatment plan. For example, imagine an organization with a high tolerance for schedule deviations but a low budget tolerance. In this case, the schedule-related risk might have an RTF of 5 , while budgetrelated risks could have an RTF of 1 .

Figure 13 illustrates the flow of risk management steps adapted to the RTF approach.

In the first stage of risk management using the RTF, two activities occur: first, there is the identification of system risks and then the determination of the RTF for each identified risk. RTF values can be determined through brainstorms, interviews with team members or experts in the field, use of questionnaires, the experience of other projects, among other ways. 
In the second stage, the identified and qualified risks are entered into the probability versus impact matrix adapted to the RTF methodology, ranging from 1 to 5, as illustrated in Fig. 14.

In the third stage of risk management, the risk mitigation plan is prepared, which will present the approach given to each risk analogous to traditional methodologies. There is often a hierarchy for risk mitigation so that those in the red region have priority and should be avoided, using techniques to eliminate the root cause of the risk, control the risk, or transfer the risk. Risks that are in the yellow region must be mitigated, having their effects on the system mitigated. The risks in the green region can be accepted, but they must likewise be monitored.

Finally, in the fourth stage, there is the action plan and risk monitoring. This plan will be passed on to other systems engineering disciplines, and the results of the risk management plan and guidelines for monitoring the risks identified in step one and parameters for identifying possible new risks are presented therein.

\section{Conclusion}

The paper has achieved its primary objective, as it presented and implemented a generic methodology for optimizing the risk management of a low-cost system with a lean schedule and short operating time.

In the analyses carried out in the two case studies of this paper, it was shown that the RTF methodology provided a smaller number of risks that should be handled in the mitigation plan. For example, in the CubeSat ARMADILLO case study, for the seven identified risks, the traditional DoD methodology and the methodology adopted for CubeSat by Yassi and Sousa produced the same approach for the identified risks. In contrast, the RTF methodology increased the risks accepted but monitored from one to three. Thus, it decreased from five to three risks that must be mitigated and maintained one risk to be avoided.

In the CubeSat CONASAT case study analysis, the traditional DoD methodology and the methodology adapted to CubeSat by Yassi and Sousa produced the same treatment for the identified risks. In the RTF methodology, the same number of risks that should be accepted was maintained, yet, when accompanied, the number of risks that must be mitigated increased from two to four, and the risks that must be avoided decreased from three to one.

Thus, when performing a proportional analysis, the CubeSat ARMADILLO case study had optimization of a $200 \%$ increase in risks that can be accepted but must be monitored and a $40 \%$ decrease in risks that must be mitigated; while, in the CubeSat CONASAT case study, optimization of a $66.7 \%$ reduction in the risks that must be avoided, and a $100 \%$ increase in the risks that must be mitigated were obtained; however, this increase is justified, as the risks that should be avoided have moved to the mitigable risks range.

\section{Declarations}




\section{Data Availability}

All data generated or analysed during this study are included in this published. Complementary information/data could be shared upon request to the corresponding author.

\section{References}

1. ECSS. EUROPEAN COOPERATION FOR SPACE STANDARDIZATION. ECSS-S-ST-00-01C. ECSS system - Glossary of terms. 2012.

2. DoD. Office of the Chairman of the Joint Chiefs of Staff, DOD Dictionary of Military and Associated Terms. Washington DC: The Joint Staff, 2021.

3. ECSS. EUROPEAN COOPERATION FOR SPACE STANDARDIZATION. ECSS-E-ST-10C. Space engineering - System engineering general requirements. 2009.

4. CGEE. CENTRO DE GESTÃO E ESTUDOS ESTRATÉGICOS - CGEE. CubeSats. Brasília, DF: 2018.

5. CAL-POLY. CDS (Rev 13). CubeSat Design Specification. 2015.

6. NASA. Improving Mission Success of CubeSats Washington: National Aeronautics and Space Administration Headquarters, 2017.

7. LUBIÁN-ARENILLAS, D.; ÁLVAREZ, J. M.;BERMEJO, J.; GARCÍA, S.; CUBAS, J.;ROIBÁS-MILLÁN, E. Nanosatellite development methodology and preliminary

8. SWEETING, M. N. Modern small satellites - changing the economics of space. Proceedings of the IEEE, v. 106, n. 3, pp. 343-361, 2018.

9. HONORÉ-LIVERMORE, E. CubeSats in University: Using Systems Engineering Tools to Improve Reviews and Knowledge Management. In: 17th Annual Conference on Systems Engineering Research (CSER). Procedia Computer Science, v. 153, pp. 63-70, 2019.

10. ALANAZI, A.; STRAUB, J. Engineering methodology for student-driven CubeSats. Aerospace, v. 6, n. 54, 2019. DOI:10.3390/aerospace6050054.

11. GAMBLE, K. B.; LIGHTSEY, E. G. Decision analysis tool for small satellite risk management. Journal of Spacecraft and Rockets, v. 53, n. 3, pp. 420-432, 2016.

12. BRUMBAUGH, K.M; LIGHTSEY, E. G. A Risk Management Plan for CubeSats. In: AIAA SPACE 2012 Conference \& Exposition. Pasadena, California, 2012. DOI: 10.2514/6.2012-5219.

13. BRUMBAUGH, K.M; LIGHTSEY, E. G. Application of Risk Management to University CubeSat Missions. JoSS, v. 2, n. 1, pp. 147-160, 2013.

14. GAMBLE, K. B.; LIGHTSEY, E. G. CubeSat mission design software tool for risk estimating relationships. Acta Astronautica, n. 102, pp. 226-240, 2014.

15. YASSI, H.; SOUSA, R. L. Developing a methodology and practical application of technical risk management for CubeSat projects. In: 6th Interplanetary CubeSat Workshop. USA: Cambridge, United Kingdom, 2017. 
16. DoD. Risk management guide for DoD acquisition. 6ed. Washington DC: Department of Defense, 2006.

17. SHAH, M. A.; KHAN, F. Y.; ASMA, R. Risk management strategy for remote sensing satellite project, following the customization of ECSS standards. Journal of Space Technology, v 1, n. 1, pp. 42-46, 2012.

18. JOHNSON, M. A.; BEAUCHAMP, P; SCHONE, H.; SHELDON, D.; FUHRMAN, L.; SULLIVAN, E.;

FAIRBANKS, T.; MOE, M.; LEITNER, J. Increase small satellite reliability - a public-private initiative. In: 31st Ann. AIAA/USU Conf. on Small Satellites. USA: Utah, 2017.

19. AHN, H.; KIM, H.; CHOI, H-L. Integrated risk management method development for multiple aerospace projects using a single index expression. International Journal of Aeronautical and Space Sciences, v. 19, pp. 1052-1062, 2018. DOI: https://doi.org/10.1007/s42405-018-0092-5.

\section{Figures}

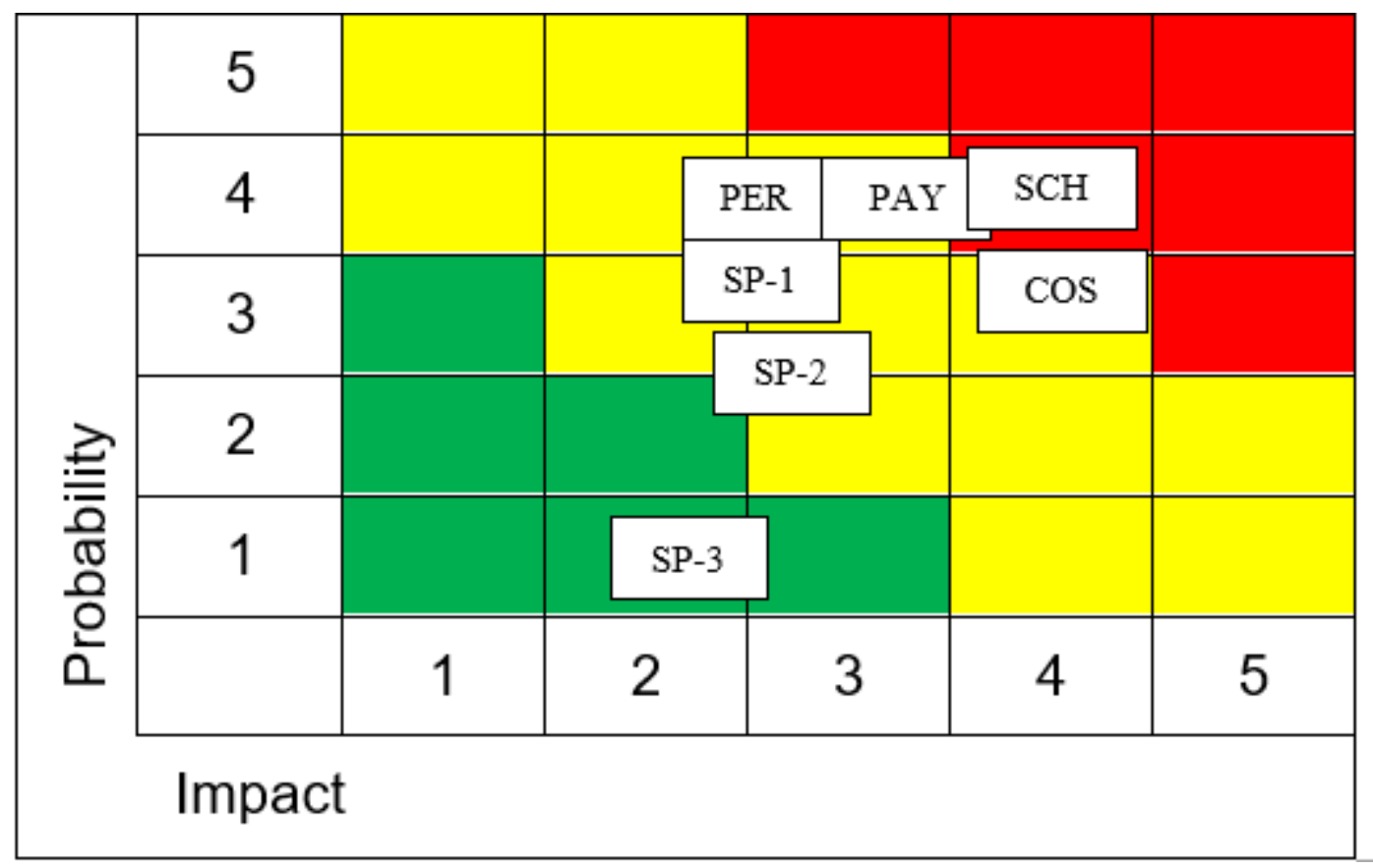

Figure 1

Probability versus impact matrix - ARMADILLO - DoD methodology 


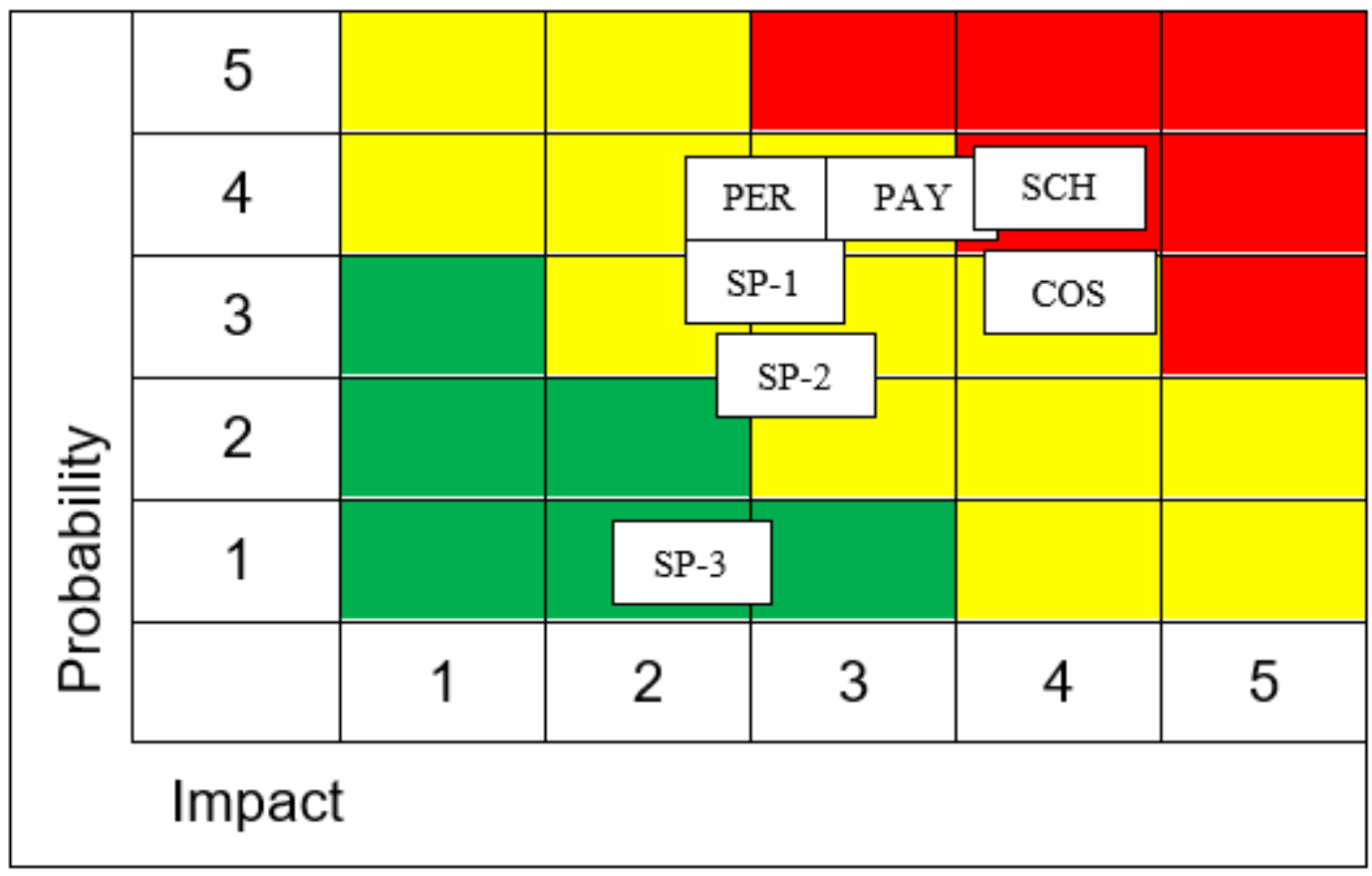

Figure 2

Probability versus impact matrix - ARMADILLO - Yassi and Sousa methodology

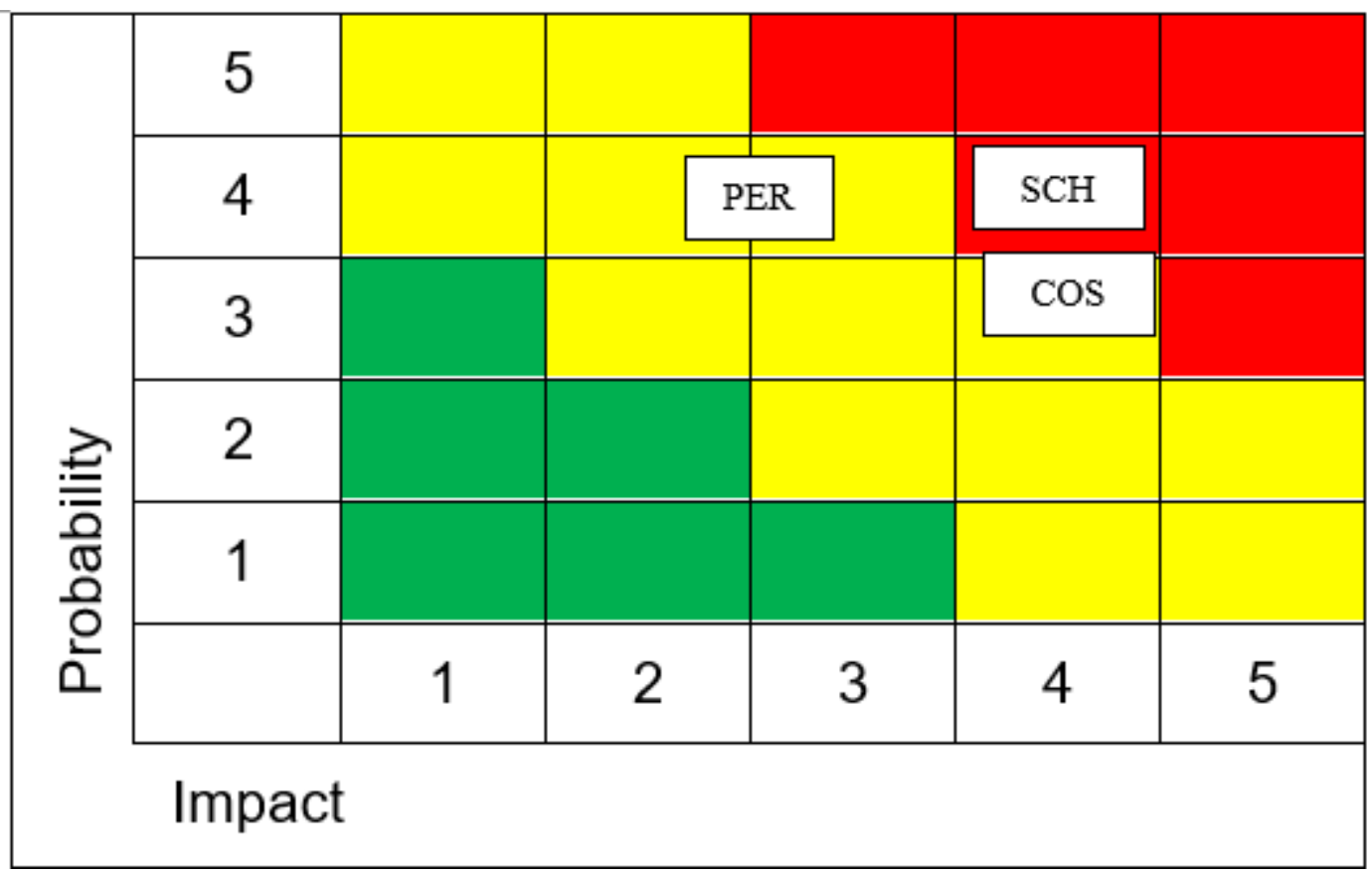

Figure 3

The ARMADILLO mission's probability versus impact matrix using RTF methodology, with RTF equal to 3 . 


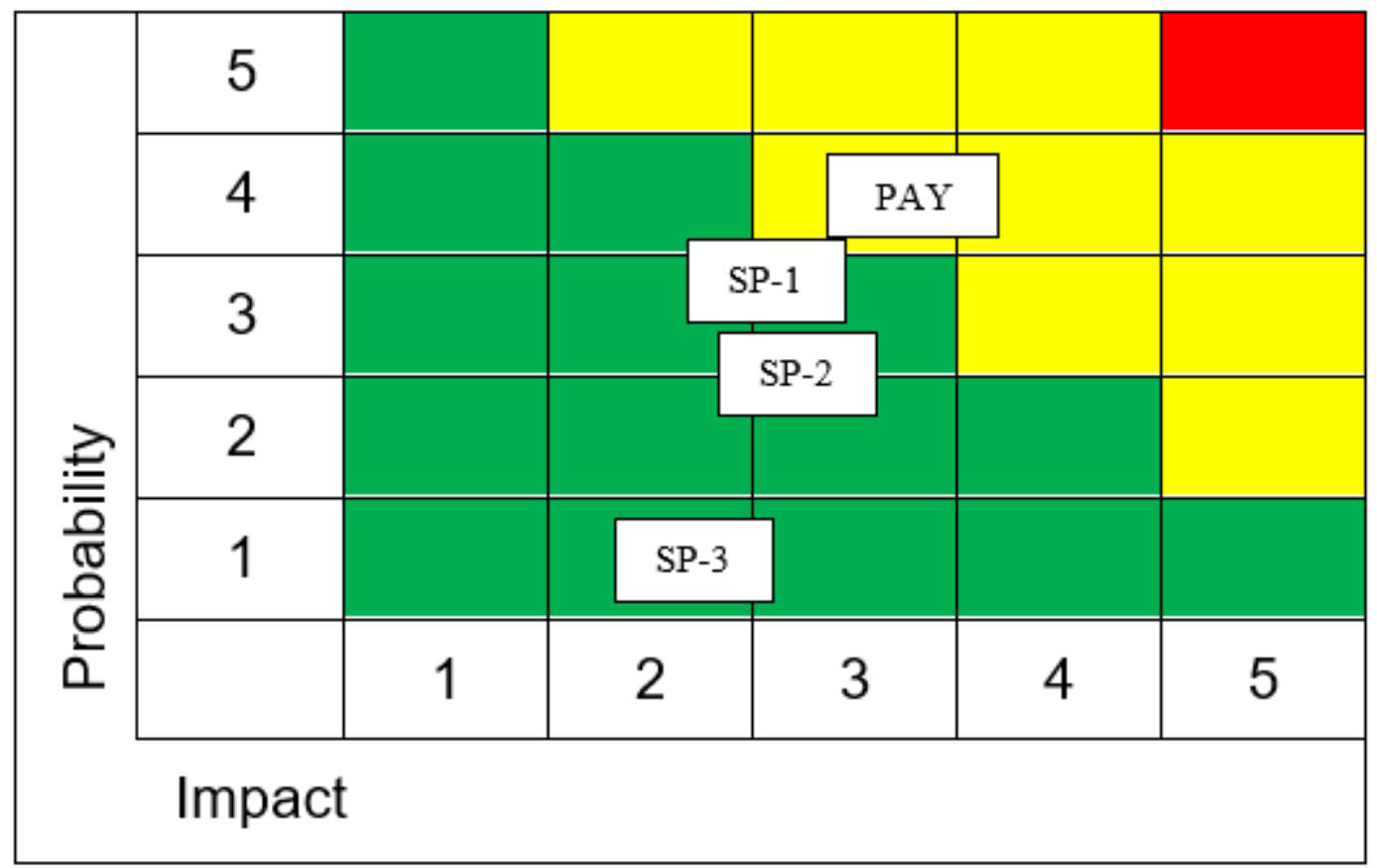

Figure 4

Probability versus impact matrix of the ARMADILLO mission using RTF methodology, with RTF equal to 5 .

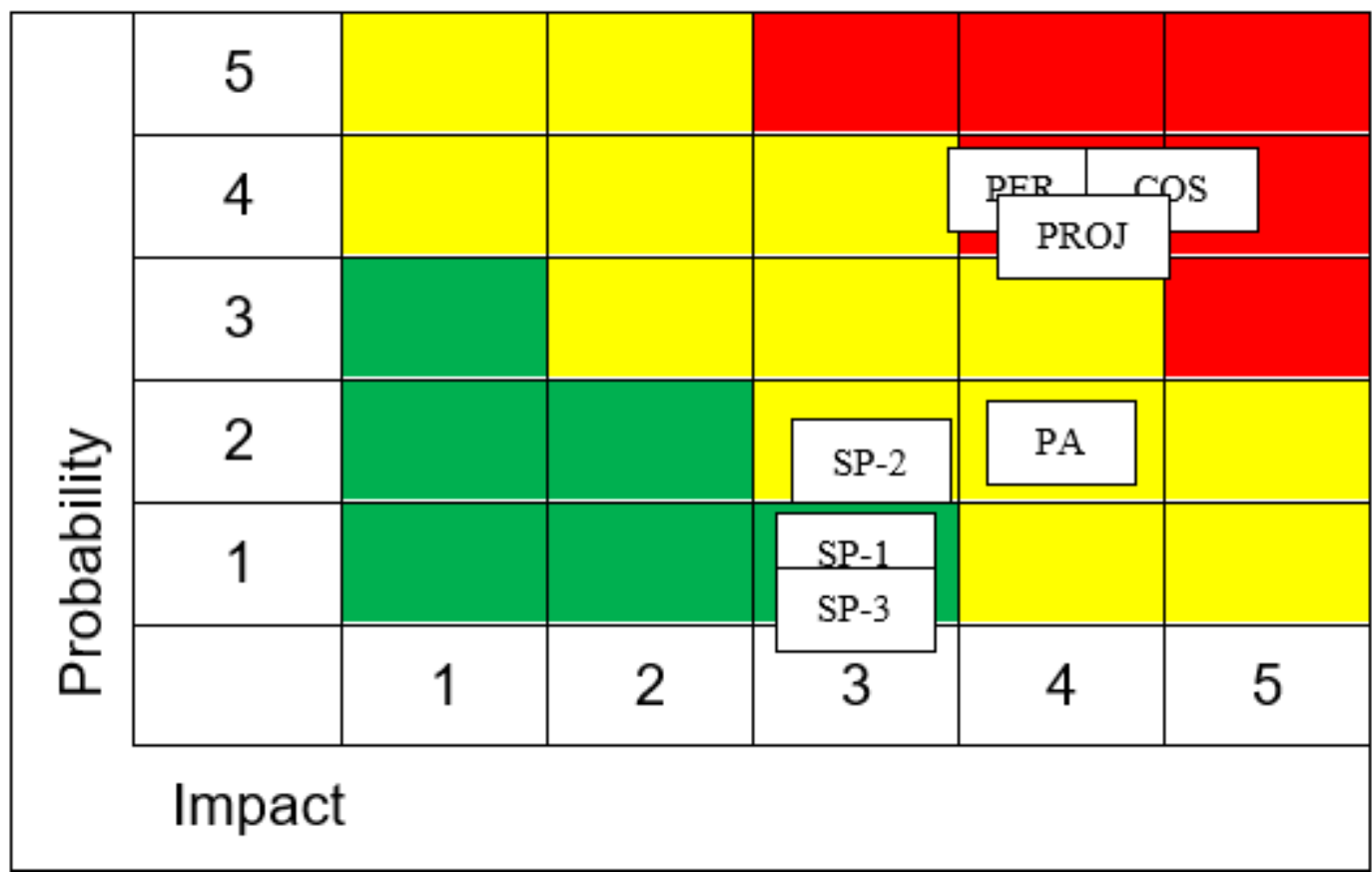

Figure 5

Probability versus impact matrix - CONASAT - DoD methodology 


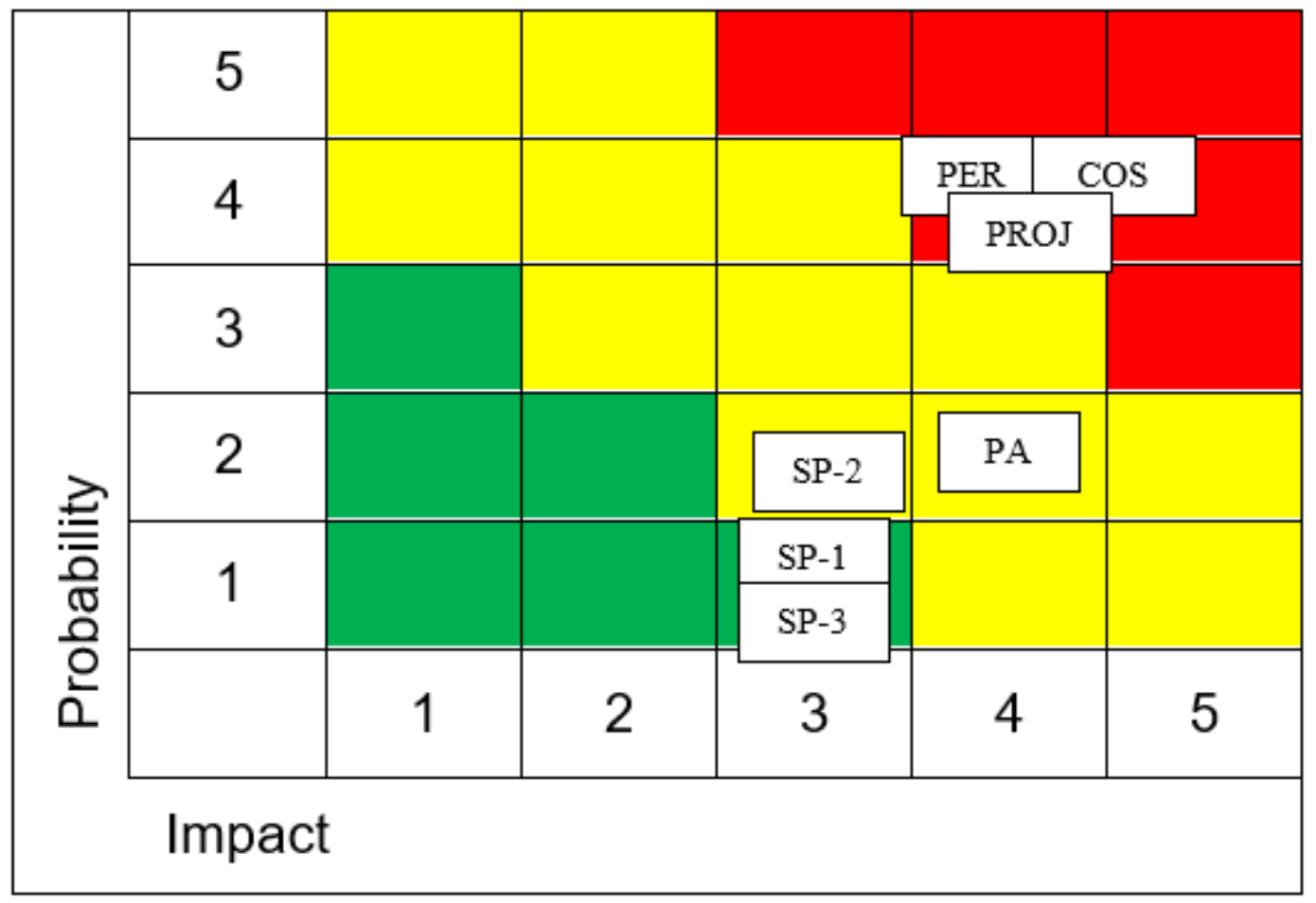

Figure 6

Probability versus impact matrix - CONASAT - Yassi and Sousa methodology

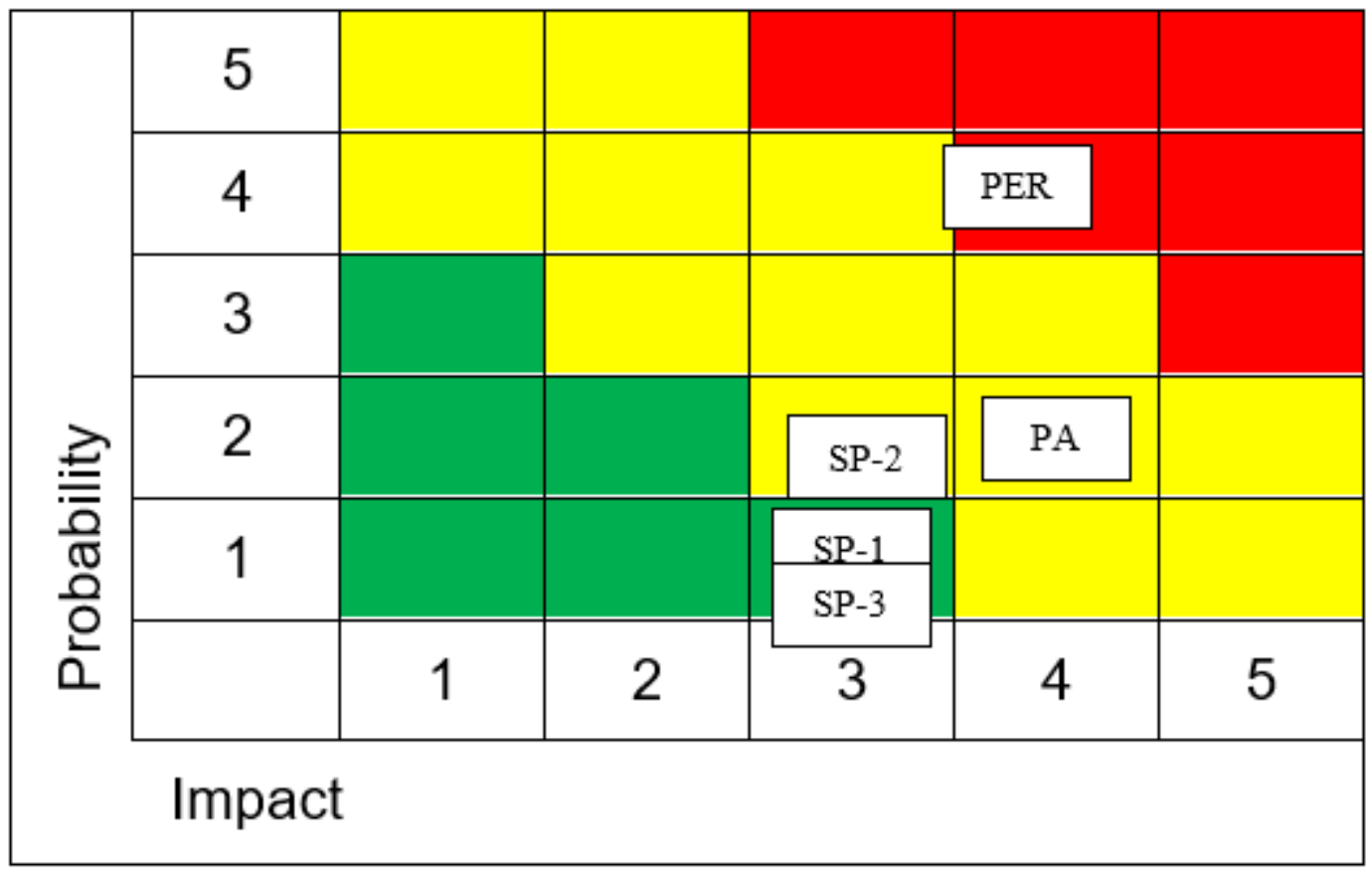

Figure 7

Probability versus impact matrix of the CONASAT mission using RTF methodology, with RTF equal to 3. 


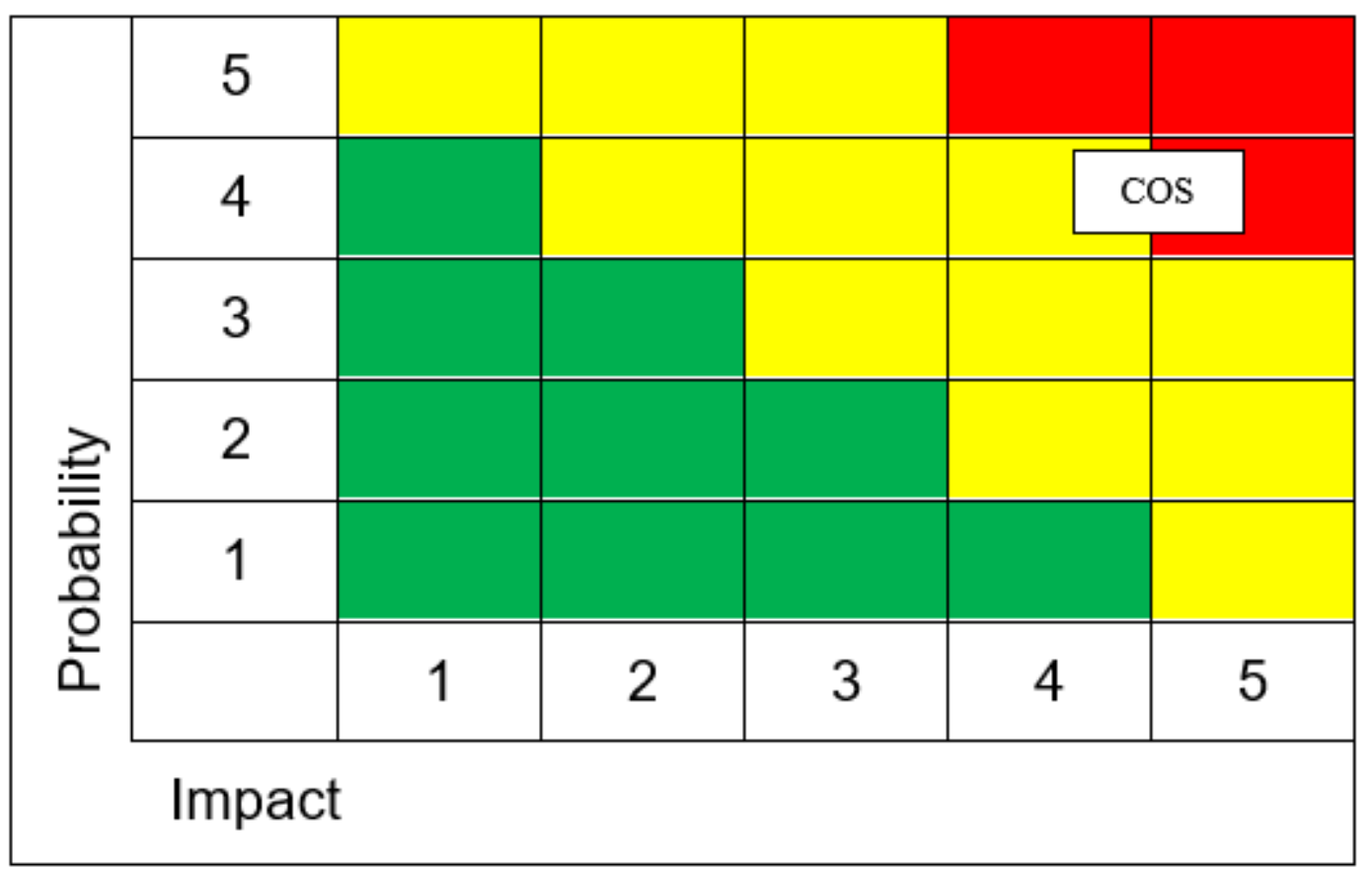

Figure 8

Probability versus impact matrix of the CONASAT mission using RTF methodology, with RTF equal to 4.

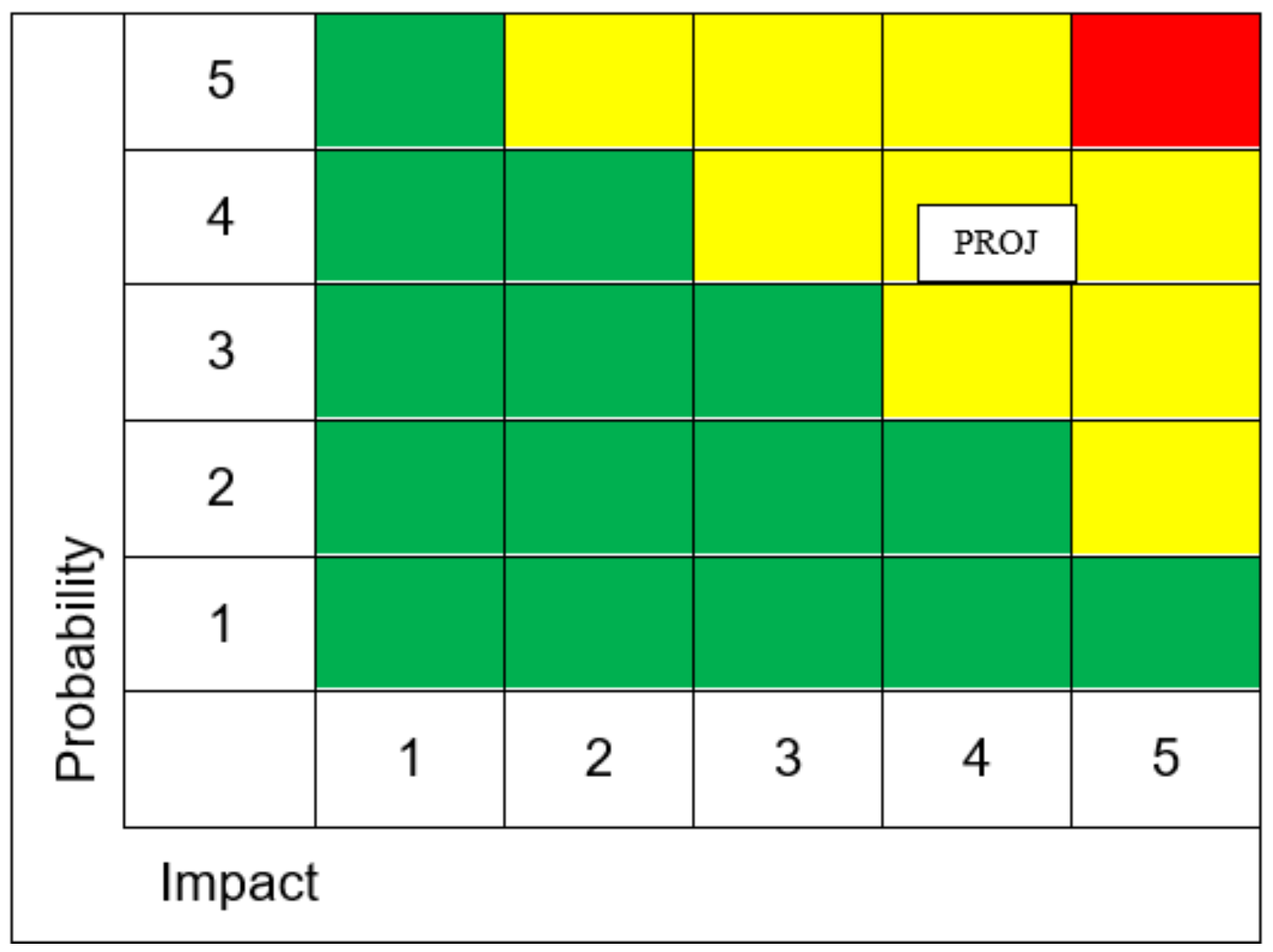

Figure 9 


\section{NASA APPROACH (DoD)}

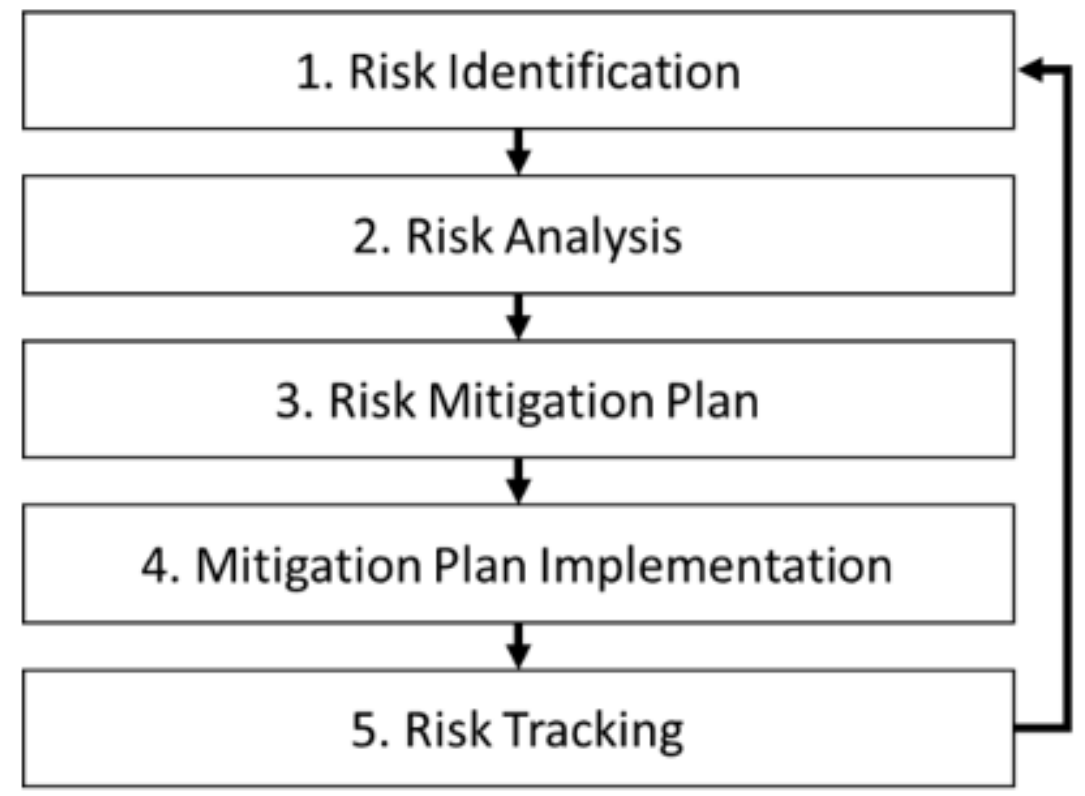

Figure 10

NASA Risk Management Flowchart (DoD) ${ }^{16}$

\begin{tabular}{|c|c|c|c|c|c|c|}
\hline \multirow{5}{*}{ 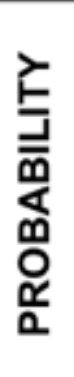 } & 5 & 1 & 2 & 3 & 3 & 3 \\
\hline & 4 & 1 & 2 & 2 & 3 & 3 \\
\hline & 3 & 1 & 2 & 2 & 2 & 3 \\
\hline & 2 & 1 & 1 & 1 & 2 & 2 \\
\hline & 1 & 1 & 1 & 1 & 1 & 2 \\
\hline & & 1 & 2 & 3 & 4 & 5 \\
\hline & & \multicolumn{5}{|c|}{ IMPACT } \\
\hline \multicolumn{2}{|c|}{ RISK } & \multicolumn{2}{|c|}{ HIGH } & MEDIUM & \multicolumn{2}{|c|}{ LOW } \\
\hline \multicolumn{2}{|c|}{ LEVEL } & \multicolumn{2}{|c|}{3} & & \multicolumn{2}{|c|}{1} \\
\hline
\end{tabular}

Figure 11

Risk Level ${ }^{15}$ 


\section{Methodology for Technical Risk \\ Management for Projects with CubeSats}

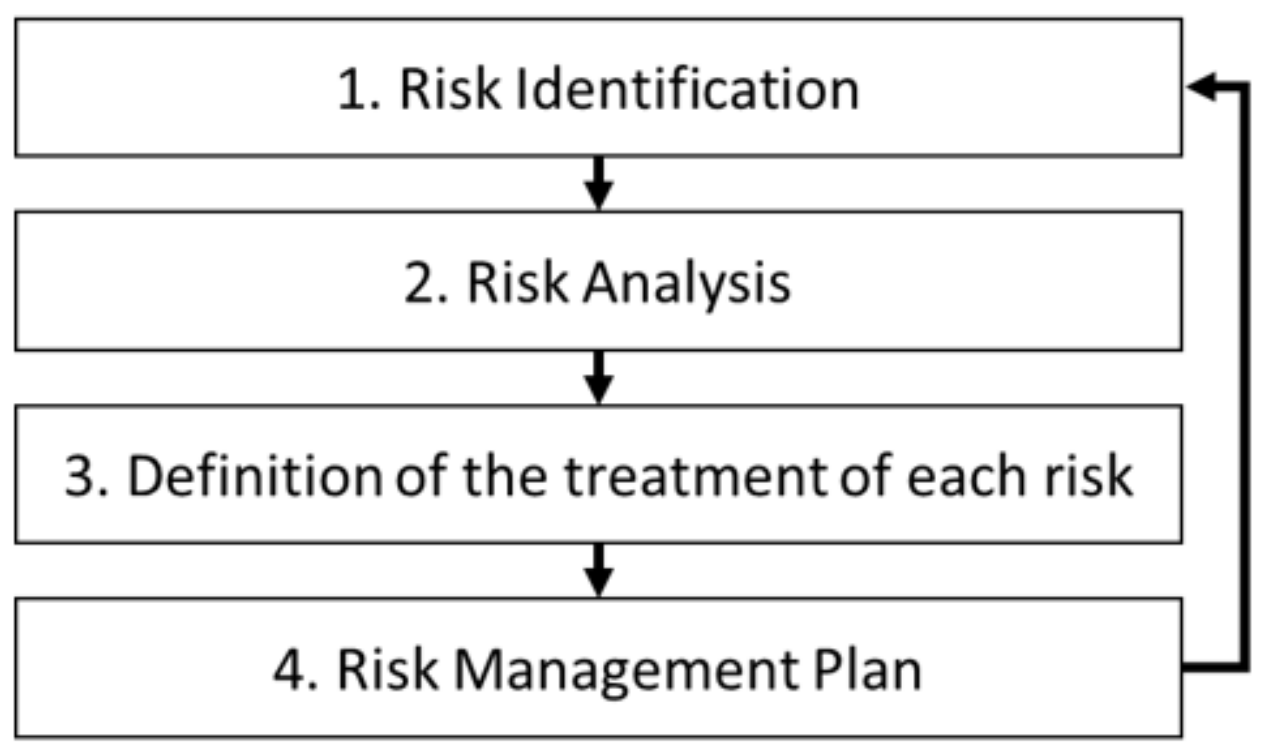

Figure 12

Flowchart Methodology for Technical Risk Management for Projects with CubeSats ${ }^{15}$.

SYSTEM REQUIREMENTS

1. RISK IDENTIFICATION

1.1 RISK IDENTIFICATION

1.2 DETERMINATION OF RFT FOR EACH IDENTIFIED RISK

2. RISK QUALIFICATION

2.1 PREPARATION OF PXI MATRIXACCORDING TO DETERMINED

RFT

$\downarrow$

3. PREPARATION OF THE RISK MITIGATION PLAN

4. RISK MONITORING AND ACTION PLAN

$\downarrow$

COST/AIT/OTHER SUBJECTS OF SYSTEMS ENGINEERING

Figure 13 


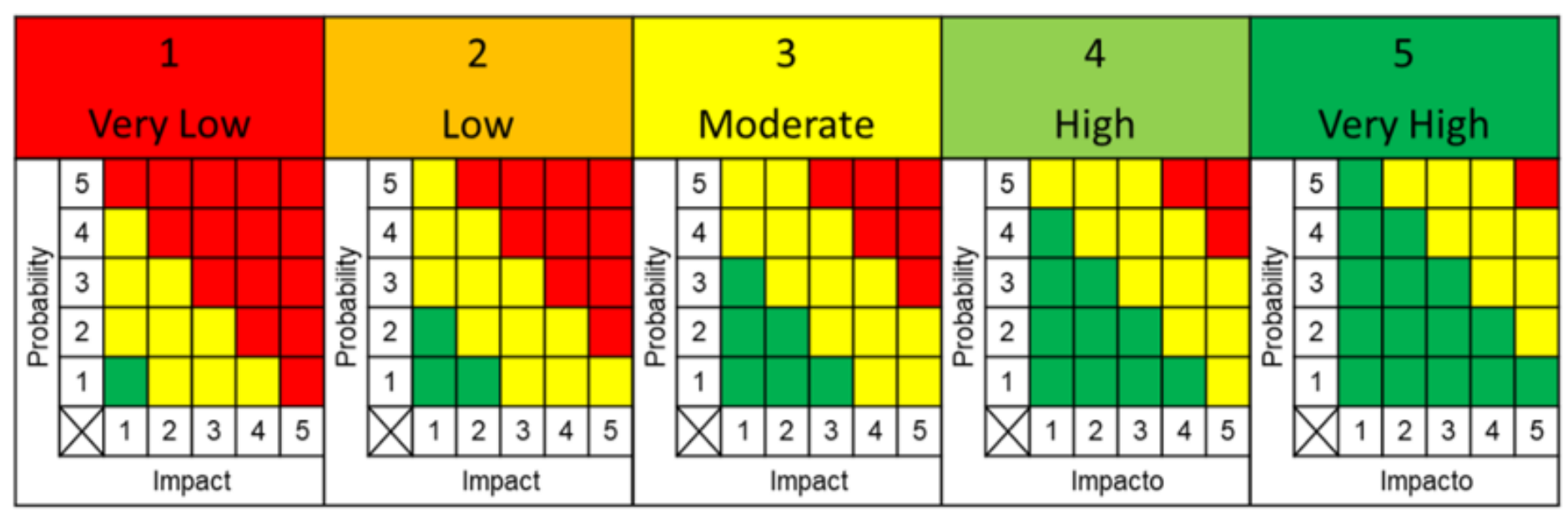

\section{Figure 14}

PxI matrices adapted to RTF methodology ranging from 1 to 5 . 